POSTGRAD. MED. J. (1965), 41, 699.

Current Survey

\title{
TORTICOLLIS
}

K. F. HulberT, F.R.C.S.

Consultant Orthopaedic Surgeon, Dartford Group Hospitals and Sydenham Children's Hospital.

Definition: A deformity, either congenital or acquired, characterised by lateral inclination of the head to the shoulder, with torsion of the neck and deviation of the face (Tubby, 1912).

Classification: Torticollis can be classified into Congenital and Acquired.

(I) Congenital Torticollis can be subdivided into:

(a) Congenital Postural Torticollis

(b) Congenital Muscular Torticollis

(II) Acquired Torticollis can be classified into:

(a) Skeletal disorders

(i) Trauma to the cervical spine, the bone, the discs or the ligaments

(ii) Inflammatory lesions of the cervical spine

(b) Neurological and psychological disorders

(i) Spasmodic torticollis

(ii) Ocular torticollis

(iii) Habit

(iv) Paralytic, peripheral or central nervous lesions

(v) Reflex, from glands in the neck

(c) Soft tissue contractions

\section{Congenital Torticollis}

Congenital torticollis is a rare deformity. Not more than three or four cases a year can be expected in an average orthopaedic clinic in this country. In one large centre 507 cases were seen over a period of 27 years, during which time 5,000 cases of club-foot were encountered (Whitman, 1927).

The congenital group of cases fall into two groups. In the first group the deformity appears at, or soon after birth, no sterno-mastoid tumour occurs and operative treatment is never required. In the second group the deformity appears some time after birth, is often associated with a sternomastoid tumour, and operation is not infrequently necessary. The first group is called postural torticollis and is temporary, the second group is called muscular torticollis and is an organic contracture.

\section{(a) Congenital Postural Torticollis}

This group comprises about two-thirds of the cases seen. The torticollis is present at birth, lasts for a few weeks, and then disappears leaving no evident contracture of the sterno-mastoid. In nearly every case there is evidence of intrauterine moulding, such as fiattening of the opposite side of the head, and occasionally deformity of the lobe of the ear on the same side. Browne (1936) described the association of postural torticollis with flattening of the opposite side of the head and moulding of the lobe of the ear against the shoulder on the same side with occasional facial paralysis on the same side.

Unless this condition is recognised and separated from the muscular group, figures relating to prognosis and treatment of the latter condition are valueless.

\section{(b) Congenital Muscular Torticollis}

Although this is the smaller of the two groups, it is by far the most famous, owing to its association with the mysterious sterno-mastoid tumour.

The natural history of this condition is that about ten days after birth a hard lump appears in the sterno-mastoid muscle, sometimes associated with a transient torticollis. The tumour usuallys disappears by the seventh month, and musculafo torticollis then develops in a proportion of cases as the short neck of the infant elongates into thae of the young child in the following two to three years. This is followed by the development of facial asymmetry and ocular disturbance due to the change in the horizon of vision.

The cause of torticollis and of the mysterious sterno-mastoid tumour has exercised the minds of surgeons for generations.

The theory of birth injury is the oldest in the field. It was thought that injury to the sternomastoid at birth resulted in the formation of a haematoma which then organised and underwent fibrous contracture. That this theory is wrong is now certain, because examination of the sternomastoid tumour in its early stage has revealed that it is a hard white fibrous tumour from the very beginning and no evidence of a haematoma in recent cases or of haemosiderin in old cases has ever been found. Of one thing we can be certain, that this is not a haematoma of the sterno-mastoid.

The theory of muscle ischaemia was first established by Nové-Josserand and Viannay (1906) who showed that the sterno-mastoid branch of the superior thyroid artery is an end artery supplying the sternal head and middle fibres of the muscle. They were then able to show that the blood flow to this portion of the muscle could be obstructed by placing the head in a position of lateral flexion. Arterial obstruction, however, produces the histological appearance of Volkmann's contracture - a massive necrosis or muscle 
slough, whereas the sterno-mastoid tumour shows an interstitial fibrosis. Middleton (1936) thought that venous occlusion produced ischaemia in muscle, and more recently Bowden and Guttman (1949) have produced ischaemia in muscle, of similar histological appearance to the sternomastoid tumour, by partial venous occlusion.

The third of the commonly held theories is that of intra-uterine malposition. Chandler and Altenberg (1944) described a case of torticollis diagnosed by ante-partum X-rays which was confirmed on delivery by Caesarean section, and in which a sterno-mastoid tumour developed on the fourteenth day. Another tumour was found at birth by Caesarean section, and yet one other in an extra-uterine gestation. The obstetric history is of great significance: in one series Hulbert (1950) showed that $40 \%$ were delivered by breech presentation, as against the over-all average of $3 \%$, and $20 \%$ were forceps deliveries. A total of $69 \%$ were abnormal deliveries.

Intra-uterine malposition is clearly a prime cause of postural torticollis. In muscular torticollis it might be postulated that the torticollis is primary, resulting in difficult labour or in the head failing to engage and thus swinging around into the breech position.

At some time the blood supply of the sternomastoid is impaired and ischaemia results. The cause must therefore be due partly to ischaemia and partly to pre-natal intra-uterine causes.

Clinical signs. The shortening of the sternomastoid muscle results in the head being laterally flexed towards the affected side, and the face being rotated towards the opposite side. In the rare bilateral case the head is extended because the sterno-mastoid is also an extensor of the occipito-atlantal joint. On palpatation the affected muscle is hard and tight. Later on, scoliosis may occur with a compensatory thoracic curve to the opposite side. Facial asymmetry has long been recognised as a complication of this condition, and patients should always be warned that one of the first effects of corrective operation is to make this appear worse. The asymmetry usually disappears within two or three years of operation, but not entirely. One aspect of the deformity, scoliosis capitis (Middleton, 1930), due to rotation of the base of the skull and deformity of the parietes, may persist. This deformity is the same as that of the thorax in scoliosis with rotation of thoracic vertebrae.

\section{Treatment}

The postural type of torticollis can be left to correct itself, aided perhaps by daily stretching of the sterno-mastoid (see below). Placing the cot so that the baby's attention is attracted by bright objects so that it turns its head in the opposite direction is worth trying.

The treatment of the muscular type is along similar lines in the first instance, and the majority of cases will yield to conservative treatment, but once an organic contracture has developed then surgical division of the tight muscle becomes necessary. The usual age for this operation is $\mathbb{D}$ two or three years, and the procedures used are $\frac{2}{c}$ either an open or a subcutaneous division of the $\stackrel{C}{-}$ sternal and clavicular heads, or a subcutaneous $\vec{F}$ division of the lower end combined with an open? division of the upper end.

The open operation. The child is anaesthetized $\frac{\overline{\bar{D}}}{\overline{\mathrm{D}}}$

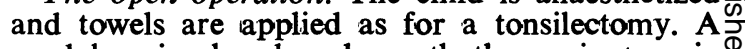
sand bag is placed underneath the occiput as in $\odot$ MacGill's position for intubation (sniffing the morning air) in order to lift the sterno-mastoid ${ }_{-}^{\infty}$ muscle forward off the great vessels lying beneath..

The skin is drawn down and incised over the $\overrightarrow{-}$ clavicle for about one-and-a-half inches. Some ${ }_{\sigma}^{\omega}$ fibres of the platysma may be divided, aftero which the outer layer of the deep cervical fascia $\frac{0}{3}$ is incised, exposing the sterno-mastoid muscle. $A \stackrel{-}{-}$ director is passed underneath, and the muscle is $A$ divided close to the clavicle. Through a smalli incision, the skin is mobile enough to allow? division piecemeal of the whole muscle with? repeated use of the director. The muscle then $₫$ retracts as the neck is stretched, revealing the? deep layer of fascia beneath which lie the greatvessels. Some surgeons divide this layer and the $z$ scalenus anterior, but this is not really necessary. After closure of the wound the head is placed in an overcorrected position until the skin Giso healed. Treatment is then as for the closed operation.

The subcutaneous operation. The scar resultikg from the open operation may be unsightly, as as this is a cosmetic operation many surgeonts prefer the subcutaneous operation, an operation which has been performed for centuries. A sharp pointed tenotome is used to puncture the sking and the superficial layer of the deep cervicalo fascia. A blunt-pointed tenotome is then passed deep to the muscle, which is then cut outwardso towards the skin laying all the time on the clavicle. If venous bleeding should occur it can be con trolled by pressure and the neck need not be opened to find the bleeding point.

The neck is then stretched to overcorrect the deformity, and the position maintained by splint-o ing. A plaster cap can be applied to the head into which flannel bandages are attached. The bandages are then strapped to the trunk to holds the head in any required position. The corrects position is that in which the mastoid and clavicle? are separated and this means flexion to the opposite side, rotating to the same side, ance. flexion of the head. After a fortnight a polythenes collar is ordered to hold the head over, and daily stretching of the neck is commenced by the physiotherapist. This continues for about three्य months and the collar is worn until the habit of
tortcollis is lost.

The combined operation. If, after having dividece the lower end of the sterno-mastoid the muscle is still tight and the organic deformity persists:then the upper end should also be divided Division of the upper end alone is not advised 
because excessive retraction of the muscle may result in paralysis of the accessory nerve. An incision is made at the level of the lower margin of the external auditory meatus, curving upwards over the mastoid and superior meatal line. The muscle is divided and erased with a rugine.

The operation, which ever is performed, is but one incident in the treatment. Unless physiotherapy is persisted with for several months and a retentive collar is worn, the condition will relapse. The child should not be discharged for at least a year.

\section{Spasmodic Torticollis}

This is a neurological disorder in which tonic and clonic contractions of the muscles of the neck are seen. It usually occurs in middle aged or elderly people, and although the aetiology is largely obscure, it may be due to degenerative changes in the basal ganglia, and it may occur as the end result of encephalitis lethargica. Conservative treatment, rest, sedatives, and splinting may be tried. The surgical treatment falls into the realm of neurosurgery and was developed by Dandy. This consists of intra-dural division of the spinal accessory nerves and the anterior roots of the 1st, 2nd and 3rd cervical nerves on both sides. Another operative measure that has been tried is complete removal of the sternomastoid muscles on the affected side.

\section{Ocular Torticollis}

Over-action of the inferior oblique muscle elevates the eye and rotates it laterally, and this action is weakest when the eye is abducted. The head is therefore turned to the opposite side in order to abduct the eye on the same side.

\section{Subluxations}

Beware of the child with a sore throat and a stiff neck. Subluxation of the axis on the atlas may complicate a simple tonsillitis, or a subluxation in the mid-cervical region may be seen after tonsilectomy under general anaesthesia. Traction applied with a head halter rapidly relieves the condition after which a cervical collar should be worn for several weeks.

In older patients the syndrome of the cervical disc may produce the same clinical picture and the treatment is essentially the same.

\section{REFERENCES}

Bowden, R. E., and Guttman, E. (1949): The Fate of Voluntary Muscle after Vascular Injury in Man, J. Bone St. Surg., 318, 355.

BrownE, D. (1936): Congenital Deformities of Mechanical Origin, Proc. roy. Soc. Med., 29, 1409.

Chandler, F. A., and Altenburg, A. (1944): Congenital Muscular Torticollis, J. Amer. med. Ass., $125,476$.

DANDY, W. E. (1930): An Operation for the Treatment of Spasmodic Torticollis, Arch. Surg., 20, 1021 .

HulberT, K. F. (1950): Congenital Torticollis, J. Bone Jt. Surg., 32-B, 50.

Middeton, D. S. (1930): The Pathology of Con genital Torticollis, Brit. J. Surg., 18, 188.

Nove-Josserand, G. and VianNaY, C. (1906): Path ogénie du Torticollis Congenital, Rev. Orthop., 25 7, 397.

TubBy, A. H. (1912): Deformities, 2nd edition, vol. i p.56. London: MacMillan.

WhITMAN, R. (1927): A Treatise on Orthopaedic Surgery, London: Henry Kimpton. 